Enfermagem Brasil 2018;17(5):552-8

\title{
RELATO DE EXPERIÊNCIA \\ Prática extensionista em grupo de idosos fundamentada na política do envelhecimento ativo
}

Daiane de Souza Fernandes, M.Sc. ${ }^{*}$, Stelacelly Coelho Toscano de Brito**, Suzayne Naiara Leal $^{* * *}$, Sara Melissa Lago Sousa ${ }^{* * *}$, Lucia Hisako Takase Gonçalves, D.Sc. ${ }^{* * * *}$

*Docente da Faculdade de Enfermagem da Universidade Federal do Pará, ${ }^{* *}$ Enfermeira, Docente na Faculdade de Enfermagem da Universidade Federal do Pará, ${ }^{* * *}$ Acadêmica do Oitavo semestre do Curso de Enfermagem da Universidade Federal do Pará, ${ }^{* * * * A c a d e ̂ m i c a ~ d o ~}$ Nono semestre do Curso de Enfermagem da Universidade Federal do Pará, ${ }^{\star \star * * *}$ Prof ${ }^{a}$ Visitante Sênior CAPES na Universidade Federal do Pará/Programa de Pós-Graduação Enfermagem

Recebido em 17 de outubro de 2017; aceito em 18 de junho de 2018.

Endereço para correspondência: Daiane de Souza Fernandes, Rua Itabira, 20 Maguari 67030-390 Ananindeua PA, E-mail: daissf@gmail.com; Stelacelly Coelho Toscano de Brito: stelacellytoscano@yahoo.com.br; Suzayne Naiara Leal: suzayneleal@hotmail.com; Sara Melissa Lago Sousa: melissalagosousa@gmail.com; Lucia Hisako Takase Gonçalves: Ihtakase@gmail.com.

\section{Resumo}

Objetivo: Descrever a experiência de desenvolvimento de educação popular em saúde dentro das práticas acadêmico-extensionista na promoção do envelhecimento ativo entre idosos usuários de unidade básica de saúde. Métodos: Relato de experiência de atividades extensionistas do projeto "Idoso Saudável" da Faculdade de Enfermagem da UFPA, iniciado em 2014 e em realização na Unidade Básica de Saúde do Guamá, Belém/PA. Resultados: A educação permanente mediada pela tecnologia de educação popular em saúde tem favorecido aprendizado dos idosos em comportamentos mais saudáveis, melhora da autoestima e maior inserção social. Conclusão: A articulação do ensino com a extensão universitária gera frutos positivos no processo ensino-aprendizagem subsidiando a formação do alunado de enfermagem ao mesmo tempo em que produz mudança comportamental dos idosos com vistas ao envelhecimento mais saudável.

Palavras-chave: idoso, relações comunidade-instituição, educação em saúde, enfermagem.

\section{Abstract}

\section{Extensionist practice in a group of elderly based on the policy of active aging}

Objective: To describe the experience of popular education development in health within academic-extensionist practices in the promotion of an active aging among elderly users of a basic health unit. Methods: Experience report of extensionist activities of the "Healthy Elderly" project of the Nursing School of UFPA, which started in 2014 and is carried out at the Basic Health Unit of Guamá, Belém/PA. Results: The permanent education mediated by popular education technology in health has favored the elderly learning of healthier behaviors, selfesteem improvement and greater social insertion. Conclusion: The articulation between teaching and university extension generates positive results in the teaching-learning process by subsidizing nursing students training produces behavioral changes in the elderly regarding a healthier aging.

Key-words: aged, community-institutional relations, health education, teaching-learning, nursing.

\section{Resumen}

Práctica extensionista en grupo de adultos mayores fundamentada en la política del envejecimiento activo

Objetivo: Describir la experiencia de desarrollo de educación popular en salud dentro de las prácticas académico-extensionistas en la promoción del envejecimiento activo entre adultos mayores usuarios de unidad básica de salud. Métodos: Relato de experiencia de actividades extensionistas del proyecto "Adulto Mayor Saludable" de la Facultad de Enfermería de la UFPA, iniciado en el 2014 y en realización en la Unidad Básica de Salud de Guamá, Belém/PA. 
Resultados: La educación permanente mediada por la tecnología de educación popular en salud ha favorecido el aprendizaje de personas mayores con comportamientos más saludables, mejora de la autoestima y mayor inserción social. Conclusión: La articulación de la enseñanza con la extensión universitaria genera frutos positivos en el proceso enseñanza-aprendizaje, subsidiando la formación del alumnado de enfermería y al mismo tiempo que produce un cambio comportamental de los mayores con el objetivo de tener un envejecimiento más saludable.

Palabras-clave: anciano, relaciones comunidad-institución, educación en salud, enfermería.

Introdução

O envelhecimento da população no Brasil vem-se apresentando de forma acelerada em consequência das mudanças sociodemográficas nas últimas décadas. De acordo com o Instituto Brasileiro de Geografia e Estatística [1], o aumento acelerado da população com idade igual e superior a 65 anos em todos os estados, correspondendo a $4,8 \%$ do total da população geral em 1991, alcançou a taxa de 7,4\% em 2010. Estima-se que em 2050 esse estrato idoso corresponda a cerca de $20 \%$ da população brasileira [2].

Considerando o atual panorama de processo de envelhecimento populacional e evidências de transição epidemiológica, a atualização e a reorganização do sistema público, social e de saúde enfrentam desafios para atender as reais necessidades de atenção aos idosos, decorrentes da prevalência de afecções crônicas mais próprias da velhice, na maioria das vezes sobrepostas, caracterizando as comorbidades. Tal condição se caracteriza como nova questão de necessidades específicas que demandam monitoramento constante do estado de saúde e de contínuo cuidado e autocuidado na família e na comunidade [3,4].

$\mathrm{O}$ aparecimento de afecções na velhice não representa necessariamente fator limitante à qualidade de vida, uma vez que ao mantê-las controladas, tratadas e cuidadas, idosos poderão levar vida autônoma, independente e produtiva dentro dos preceitos político-sociais de determinantes do envelhecimento ativo [3,5].

$\mathrm{O}$ atual perfil característico dos idosos em nosso meio, principalmente daqueles residentes em periferias empobrecidas de grandes cidades [6,7], induz-nos à necessidade de redirecionar a capacitação de enfermeiras(os) e do ensino de acadêmicos de enfermagem, focando $o$ atendimento de pessoas idosas com perspectiva multissetorial. Tal abordagem se constitui em desafios a serem enfrentados, pois devem ser encontradas tecnologias educacionais operativas voltadas para a gerontocultura, enfatizando, dentro dos aspectos éticos do cuidado, as práticas de promoção e manutenção do envelhecimento ativo e saudável.

Nessa circunstância, como enfermeira na função docente universitária na UFPA/FAENF, assumimos, em conjunto com os pares docentes, os profissionais de serviço e, necessariamente, os acadêmicos de enfermagem, o compromisso de desenvolver estratégias de educação popular em saúde com o intuito de cultivar o envelhecimento ativo com base na prática extensionista universitária, protocolada sob o título "Idoso Saudável", cujas atividades se iniciaram em 2014 com um grupo de usuários idosos na Unidade Básica de Saúde de Guamá, Belém/PA.

Do aspecto de prática educacional para formação dos acadêmicos, foram enfatizadas as ações com visão humanística, crítica e reflexiva, com o intuito de torná-los capazes de atuar com responsabilidade social de compromisso com a cidadania de cada um dos idosos participantes do programa de extensão.

A formação do enfermeiro torna-se fundamental quando incentivado a desenvolver aprendizagem vivencial sob a forma de extensão [8,9], inclusa na tríade das funções universitárias - ensino, pesquisa e extensão - na qual se supõe ensino transversal do projeto político-pedagógico do currículo acadêmico [10], favorecendo a formação de perfil profissional que atuará dentro da atual conjuntura demográfico-epidemiológica do envelhecimento no país, o que requer o enfrentamento de novos desafios de atenção à população idosa, tanto no âmbito individual como coletivo.

Neste sentido, o objetivo do presente artigo é relatar a experiência de desenvolvimento de ações de educação popular em saúde dentro das práticas acadêmico-extensionistas na promoção do envelhecimento ativo entre idosos usuários de uma Unidade Básica de Saúde, destacando a contribuição da aprendizagem vivencial para o empoderamento pessoal e coletivo dos atores: os usuários idosos, os acadêmicos de enfermagem e os profissionais envolvidos. 
Nunca na história da humanidade se observou o fenômeno do aumento da população idosa e o alongado de sua longevidade. $O$ censo demográfico brasileiro tem demonstrado que a cada período censitário o estrato idoso tem crescido proporcionalmente, além do aumento, em número absoluto, de idosos mais idosos - aqueles com 80 anos ou mais [1]. Tal fenômeno reflete a conquista social de políticas econômicas e sociais da segunda metade do século XX, resultando na melhoria das condições de vida [11]. Contudo, o prolongamento da vida humana requer investimentos pessoais de quem envelhece, exigindo educação permanente para a vida, para que a longevidade das pessoas não seja apenas o mero acréscimo de anos à vida, mas de vida mais longa com qualidade, como propõe a OMS [5]. As pessoas nunca viveram tanto e ao mesmo tempo nunca sentiram tanta necessidade de aprender o que fazer e como viver o tempo que thes é dado. Daí a existência de universidades da terceira idade e grupos de convivência repletos de idosos, em quase todas as regiões do país [12].

Assim, a educação permanente para a vida nunca foi tão necessária como hoje, para capacitar seres humanos para protagonizar seu próprio desenvolvimento, permitindo que cada um se emancipe e se torne um cidadão que reconhece seus direitos e deveres, além de compromissos consigo mesmo e com a sociedade [13]. Essa educação se torna requisito importante para a vida das pessoas, fazendo-as participar ativamente da sociedade. Por meio de tecnologias ativas de educação em processos de vida e saúde, podem ser abordadas temáticas várias de cultivo do bem viver na velhice, atuando em consonância com o que é estabelecido na Política Nacional de Saúde da Pessoa Idosa e na Política do Envelhecimento Ativo.

\section{A política do envelhecimento ativo - um marco de referência para a prática extensionista}

O envelhecimento ativo é uma política de otimização das oportunidades de saúde, educação, trabalho, participação social e segurança, com o propósito de promover o bem-estar e a melhoria da qualidade de vida das pessoas, durante todo o processo de envelhecimento, possibilitando a vivência da longevidade mais saudável possível; e quando, por circunstâncias várias, levarem pessoas ao comprometimento da vida cotidiana, com perda da autonomia e independência, Ihes sejam disponíveis cuidados condignos por toda a vida, como direito e solidariedade humana $[5,14,15]$.

A Organização Mundial de Saúde [5] define política do envelhecimento ativo como o processo de prover e oportunizar às pessoas idosas serviços sociais e de saúde e participação social e segurança para garantir-lhes um envelhecimento com a maior qualidade possível. Tal conceito pressupõe que as pessoas sejam induzidas a perceber o seu potencial para buscar o bem-estar biopsicossocial e espiritual ao longo da vida. O termo "ativo" refere-se à participação social, econômica, cultural, espiritual e civil de forma contínua, e não apenas à capacidade física da pessoa ou de pertencer à força de trabalho na sociedade. Todas as pessoas em processo de envelhecimento ou que apresentem alguma afecção, geralmente crônica, podem ainda continuar contribuindo de forma positiva e ativa para seus familiares, sua comunidade e seu país. A política do envelhecer ativo decorre do aumento da expectativa de vida das pessoas. $\mathrm{O}$ indivíduo pode envelhecer ativamente ao reconhecer seus direitos humanos e os princípios de autonomia e independência, de participação social, de atenção condigna e de autorrealização, estabelecidos pela Organização das Nações Unidas. Essa abordagem deixa de considerar os idosos como passivos e passa a considerá-los como cidadãos, que reconhecem seus direitos e deveres e buscam a igualdade de oportunidades e tratamento em todos os aspectos da vida à medida que envelhecem. Para que o envelhecimento ativo se estabeleça entre as pessoas é importante que as políticas e programas locais tenham potencial para envolver toda a comunidade; e que setores de políticas sociais, de saúde, de mercado de emprego e trabalho e de educação promovam e apoiem a busca pelo envelhecimento ativo para toda a população. Para que as políticas e programas destinados ao envelhecimento ativo tenham êxito é necessário que seus "fatores determinantes" sejam considerados prioritariamente com inclusão de insumos para melhorar as condições gerais e para o usufruto da coletividade, como de programas educacionais, de serviços sociais e de saúde, de moradia condigna para as pessoas em geral, para as famílias e para a comunidade como um todo. Os fatores determinantes, como: os econômicos, sociais, comportamentais, pessoais, de serviços sociais e de saúde e ambiente físico podem interagir entre si e inclusive entre a cultura e as questões de gênero; são fatores essenciais que contribuem para que as populações envelheçam de modo ativo e saudável $[5,15]$. 
Contribuir na promoção de envelhecimento ativo por meio de práticas acadêmicas extensionistas de enfermagem com métodos de educação popular em saúde requer principalmente o reconhecimento dos direitos de cidadania da pessoa idosa, assim como a compreensão dos princípios de participação social, autorrealização e assistência condigna, representativos de conceitos primordiais para direcionar uma abordagem que permita 0 reconhecimento dos direitos dos mais velhos e a igualdade nas oportunidades e atenção em todos os aspectos da vida.

\section{Métodologia}

O presente relato de experiência de atividades extensionistas, desenvolvidas por meio de processo educativo, é parte do projeto "Idoso Saudável" da Faculdade de Enfermagem da UFPA. Criado em 2014, suas atividades realizam-se mensalmente na Unidade Básica de Saúde do Guamá, onde esses encontros proporcionam trabalhar práticas que proporcione aos idosos envelhecimento ativo e conhecimento sobre os determinantes sociais de saúde, situados em um distrito de periferia empobrecida do município de Belém/PA, campo oficial de prática do alunado da área da saúde da UFPA.

Para implantar e consolidar esse projeto de extensão, foram realizadas atividades de formação e capacitação do grupo (estudantes de enfermagem, docentes de enfermagem e enfermeiros voluntários), além de rastreamento e cadastro dos idosos usuários da Unidade interessados em participar do projeto. No formulário de cadastramento foram incluídos: dados de identificação como as variáveis sociodemográficas; antecedentes familiares e pessoais; dados de saúde, como: história de comorbidades, tratamentos e controle; comportamentos de vida e saúde; funcionalidade para atividades da vida diária e outros dados constantes da Caderneta do Idoso.

Dirigido aos idosos usuários de Atenção Primária do sistema SUS, o "Projeto Idoso Saudável" visa à prática do envelhecimento ativo, adotando uma estratégia educacional adaptada ao público gerontológico de convivência grupal, com o alunado jovem de enfermagem, com os profissionais e docentes envolvidos, além dos demais voluntários, todos em convívio dialógico horizontal e compartilhamento de experiências entre diferentes gerações [16].

Esse projeto de extensão teve origem nas necessidades identificadas no ensino curricular formal de "Atenção Integral à Saúde do Adulto e Idoso" do terceiro semestre do Curso de Enfermagem da UFPA para produzir integração mais consistente entre ensino e assistência para o estrato idoso da população, cada vez mais presente nas Unidades Básicas de Saúde. Com essa perspectiva, o envolvimento do alunado de enfermagem em práticas extensionistas como parte de atividade curricular busca aprendizagem efetiva em ação real conjunta com os usuários idosos, atores desejosos de ver atendidas suas necessidades de vida e saúde. Sua elaboração e implementação - uma estratégia dinâmica conjunta com idosos - resultam em eleição de questões temáticas de saúde de maior importância para o grupo e seleção de tecnologias cuidativo-educacionais mais apropriadas para discutir comportamentos mais saudáveis com vistas a conquistar o envelhecimento ativo.

Considerando ser essa Extensão Universitária um projeto contínuo, ano após ano, são introduzidas atualizações, instruções e rememoração das ações do projeto realizadas periodicamente com a equipe participante atual, incluindo não apenas o grupo de usuários idosos participantes, grupo esse já acostumado a sofrer exclusões, mas sobretudo incluir novos idosos.

\section{Resultados}

Identificação dos idosos participantes e dinâmicas das atividades de encontros vivenciais

O presente projeto de extensão tem como público alvo os usuários idosos de 60 e mais anos de idade com disponibilidade para participar mensalmente de encontros vivenciais. Atualmente, o projeto conta com mais de 90 idosos cadastrados, que participam efetivamente, sendo $89 \%$ do sexo feminino e $11 \%$ do sexo masculino. Convém salientar que esse quantitativo de participantes se justifica pelas dificuldades pessoais de cada idoso em comparecer à unidade, tanto por difícil acessibilidade urbana, quanto por problemas de saúde que o impedem de sair de casa. 
Nos encontros vivenciais, desenvolvem-se não apenas metodologia participativa de educação popular versando sobre a questão de saúde e envelhecimento previamente eleita pelo grupo, mas também temas importantes veiculados pela mídia e que inquietam os idosos, discutindo-os sob a óptica de conhecimentos gerontológicos, partilhando experiências, questionando e encontrando melhores posicionamentos pessoais e grupais no enfrentamento de questões em pauta.

As mais variadas atividades são trabalhadas nas abordagens temáticas permeando ações complexas que atendam às necessidades físicas, psicossociais e educacionais voltadas para o autocuidado na terceira idade (individual e de família), negociado para estabelecer vínculos na rede de suporte social, resgatando experiências e valorizando saberes populares e técnicos facilitadores do cuidado de outrem, em situações de idosos que necessitam de cuidadores.

O espaço criado pelo projeto "Idoso Saudável" tem possibilitado aos idosos um ambiente de convivência propício para que eles possam empoderar-se e participar ativamente em atividades que gerem maior qualidade de vida e produtividade pessoal e social.

\section{Experiência de atividade extensionista na provisão de ensino transformador na enfermagem}

As evidências de envelhecimento das pessoas no país são cada vez mais nítidas, conforme os índices apresentados pela estatística nacional [2]. A proposta político-pedagógica da UFPA/FAENF em relação à realidade atual chama a atenção para a necessidade de serem produzidas mudanças na formação diferenciada de futuros enfermeiros. Com vistas a tal desafio, a equipe de docentes de enfermagem do 3ำ período de Graduação de Enfermagem, no qual se aloca o ensino da enfermagem ao adulto e a enfermagem gerontológica, lançou um projeto de experimentação de atividades de extensão atreladas aos ditames universitários do tripé - ensino, pesquisa e extensão - na formação do enfermeiro com novo perfil, capaz de empreender mudanças sociais necessárias.

A extensão universitária, conforme temos realizado, é um processo de cunho técnicocientífico, educacional e social, no qual são desenvolvidos conhecimentos diferenciados, com uma vertente transformadora, tanto no âmbito da universidade como da comunidade, por se fundamentar na troca de saberes acadêmicos e populares. Tratando-se de uma extensão universitária democrática, busca integrar teoria e prática - prática esta geralmente interdisciplinar - possibilitando sua avaliação pela comunidade envolvida e pela sociedade em geral. De concepção freiriana, a extensão universitária é tida como um processo dialético, pela valorização e conhecimento do outro com intuito basilar de desenvolver práticas transformadoras, atrelando o cuidado e a educação em saúde, caracterizando elo indissociável na formação norteadora das práticas extensionistas [17].

Atividades que englobam o ensino e a extensão se revestem de extrema importância, tanto no âmbito acadêmico quanto societal, conforme se observou ao longo dos 3 anos de funcionamento. Trabalhar com questões gerontogeriátricas é um desafio, pois envolve abordagens teóricas atreladas às práticas extensionistas direcionadas às atividades de educação em saúde, culminando com integralidade assistencial com reflexos diretos na prática cotidiana das pessoas idosas.

Nessa perspectiva, envolver o alunado de enfermagem na integração de atividade curricular com prática de extensão enseja-Ihe maior envolvimento no processo de educação e assistência na atenção integral ao idoso, pela percepção proporcionada acerca do processo de cuidar de forma global e multiprofissional, refletindo melhor a prestação de uma assistência mais completa ao usuário.

Segundo escritos sobre extensão com foco na pessoa idosa, nas universidades brasileiras, teve seu auge no início século XXI. Nesse aspecto, as pessoas idosas, geralmente de camadas menos privilegiadas, participam de programas comunitários com base social, cultural e educativa, atrelados concomitantemente ao processo dinâmico de ensino, pesquisa cultural e extensão. Assim, essas vivências proporcionam um retorno de aprendizado na velhice por meio de (re)construções de experiências individuais e coletivas. $\mathrm{O}$ envolvimento em questões de envelhecimento incitam inquietações que mantêm os idosos ativos em programas de extensão, onde atividades os envolvem em uma nova perspectiva de resgate sociopessoal [8].

Dessa forma, o propósito do projeto "Idoso Saudável", que é proporcionar o desenvolvimento do envelhecimento ativo, vem contribuindo efetivamente para esse processo, por meio de uma educação adaptada ao público gerontológico e, ao mesmo tempo, a 
possibilidade de conviver com o meio acadêmico, fortalecendo assim o partilhar de experiências tanto entre diferentes gerações quanto de saberes técnicos e populares.

Dentre os projetos de extensão desenvolvidos pela UFPA, destaca-se em especial seu modo paralelo à proposta pedagógica da Enfermagem, nas vertentes de formação de bacharelado e de licenciatura [10]. Assim, busca-se aqui, executar a prática em analogia ao do processo de educar, onde o idoso apoiado em processo de seu empoderamento, torna-se protagonista da construção do seu próprio envelhecer com qualidade. A extensão universitária potencializa as práticas educativas para proporcionar ao idoso, familiares e à comunidade 0 conhecimento multidisciplinar, através de troca de experiências, viabilizando o processo de ensino-aprendizagem na busca por transformações positivas no processo de construção e reconstrução de saberes em contexto real [9].

Convém salientar a repercussão dos efeitos do grupo de extensão em estudo, quando foi convidado a estabelecer integração numa comunidade vizinha denominada Monte Serrat, onde se buscou ampliar as discussões com outras pessoas idosas que ansiavam partilhar experiências e desenvolver o autoconhecimento, discutindo e aprendendo o protagonismo do próprio envelhecer com mais qualidade, por meio da educação popular em saúde, meta do projeto "Idoso Saudável".

Logo se percebe que a relação do idoso com o projeto de extensão se concretiza com a criação de relacionamentos seguros oportunizados na construção da autopercepção do idoso na sociedade, expressada por atitudes de autoconfiança, vínculo entre todos os integrantes do projeto, bem como participação ativa em atividades de grupo, com proposição de ações e compartilhamento de experiências pessoais, dentro dos temas de vida e saúde abordados.

A repercussão do compartilhamento de notícias pela ferramenta digital na divulgação das atividades realizadas mensalmente com os idosos, valorizando seus feitos, e a comunicação de informações pertinentes aos conhecimentos de gerontogeriatria da página do Projeto na mídia social Facebook, permitiram ampliar e divulgar entre a comunidade local a importância de ser repassada a proposição do Projeto e disseminá-lo para tornar-se conhecido, evidenciando a real relevância da vivência para o envelhecimento ativo, como direito de cidadania, e até mesmo para divulgar o que muitos desconhecem acerca de envelhecimento, de velhice e de pessoas idosas.

A extensão universitária possibilita aos acadêmicos o repasse de conhecimentos técnico-científicos adquiridos na universidade, de modo articulado e congruente com o saber popular, ao atender as demandas do público alvo, resignificando a relação ensinoaprendizagem de forma dinâmica, conduzindo a mudanças possíveis [9]. Os acadêmicos de enfermagem passam a ter uma visão diferenciada do plano de intervenção, valorizando o contexto real e os saberes dos atores participantes.

Conclusão

A busca por (re)construção de conhecimentos e comportamentos de vida e saúde voltados para o envelhecimento ativo se pauta na interação da tríade universitária - ensino, pesquisa e extensão - na qual a articulação do ensino com a extensão seja enfática na educação permanente para o desenvolvimento humano, mediada por tecnologias de educação popular para o cultivo protagonista do bem viver dos envelhecentes, os idosos usuários do sistema SUS, em postura empoderada de cidadãos ativos e cientes dos seus direitos e deveres humanos.

Desempenhado pelo enfermeiro de serviço e ou pelo docente universitário, o papel de educador em enfermagem inclui funções diretas de responsabilidade nesse processo de educação realística para a vida dos usuários, quando conseguem trabalhar na dialogicidade horizontal e encontrar juntos os melhores direcionamentos da vida, sejam pessoal ou coletivo.

$\mathrm{Na}$ perspectiva aqui analisada, o envolvimento dos acadêmicos de enfermagem na prática extensionista oportuniza reflexão e posicionamento sobre a multifacetada realidade da pessoa idosa e do envelhecer humano e sobre o seu papel social enquanto futuro profissional.

A educação permanente para o cultivo do próprio envelhecimento como usufruto no desenvolvimento humano enquanto pessoas que envelhecem é parte fundamental a ser mantida e estimulada para o desenvolver positivo das pessoas idosas. Tal ação educacional, por sua vez, influencia diretamente a capacitação dos profissionais e acadêmicos para lidar adequadamente com a temática do envelhecimento humano.

O envolvimento do alunado de enfermagem nas práticas extensionistas, desfrutando de ações cuidativo-educacionais no real campo de prática acadêmica, como tem ocorrido no 
desenvolvimento do presente projeto "Idoso Saudavel", refletirá certamente na formação desses futuros profissionais enfermeiros com visão e postura diferenciadas na atenção da população idosa nos domínios teórico, prático e político, valorizando a pessoa idosa em particular e a população idosa em geral.

\section{Referências}

1. IBGE. Instituto Brasileiro de Geografia e Estatística. Censo 2010. [citado 2016 Abr 24]. Disponível em: http://censo2010.ibge.gov.br.

2. IBGE. Instituto Brasileiro de Geografia e Estatística. Projeção da população por sexo e idade para o período de 2000/2060; Projeção da população das unidades da federação por sexo e idade para o período 2000/2030. Rio de Janeiro, 2013. [citado 2016 Nov 11]. Disponível em:

http://ftp.ibge.gov.br/Projecao_da_Populacao/Projecao_da_Populacao_2013/nota_met odologica_2013.pdf.

3. Brito MCC, Freitas CASL, Mesquita KO, Lima G.K. Envelhecimento populacional e os desafios para a saúde pública: análise da produção científica. Revista Kairós Gerontologia 2013;16(3):161-78.

4. Miranda GMD, Mendes ACG, Silva ALA. O envelhecimento populacional brasileiro: desafios e consequências sociais atuais e futuras. Rev Bras Geriatr Gerontol 2016;19(3):507-19.

5. OMS. Organização Mundial de Saúde. Envelhecimento ativo: uma política de saúde. Tradução de Suzana Gontijo. Brasília: Organização Pan-americana da Saúde; 2005.

6. Fernandes DS. Avaliação da capacidade funcional de idosos longevos amazônidas [Dissertação]. Belém: Programa de Mestrado em Enfermagem da Universidade do Estado do Pará - UEPA; 2015.

7. Gonçalves LHT, Polaro SHI, Carvalho JN, Góes TM, Medeiros HP, Souza FJD. Condições de vida e saúde de idosos amazônicas: realidade de comunidades periféricas de cidades paraenses. Rev Enferm UFPE 2015;9:321-7.

8. Almeida FS, Silva FEA. Extensão universitária para idosos: um espaço de construção do saber. In: VII Jornada Internacional de Políticas Públicas. São Luís: UFMA; 2015.

9. Pasquim EM, Recine E. Contribuições da extensão universitária para a construção de conhecimento em segurança alimentar e nutricional. Demetra: alimentação, nutrição \& saúde 2017;12(3):539-59.

10. Universidade Federal do Pará (UFPA) - Instituto de Ciências da Saúde: Faculdade de Enfermagem. Projeto Político-pedagógico do Curso de Enfermagem. Belém: UFPA. 2008. 88p (documento interno).

11. Camarano AA, Kanso S. Envelhecimento da população Brasileira - uma contribuição demográfica. In: Freitas E. et al. Tratado de geriatria e gerontologia. $4^{\underline{a}}$ ed. Rio de Janeiro (RJ): Guanabara-Koogan; 2016. p. 58-73.

12. Cachioni M. Universidade da terceira idade: história e pesquisa. Rev Temática Kairós Gerontologia 2012;5;1-5.

13. Delores SJ (org.). Educação: um tesouro a descobrir. Relatório da Unesco da Comissão Internacional Sobre Educação para o século XXI. Organização das Nações Unidas para educação, a ciência e a cultura. Ed. CNPq/IBICT/UNESCO [internet]. Brasília (DF): Unesco; 2010 [citado 2014 out 10]. Disponível em: http://unesdoc.unesco.org/images/0010/001095/109590por.pdf

14. Brasil. Ministério da Saúde (MS). Cadernos de atenção básica. Envelhecimento e saúde da pessoa idosa. Brasília: MS; 2006. 192 p.

15. OMS. Organização Mundial de Saúde. Guia Global das Cidades Amigas do Idoso. Traduzido do inglês. Lisboa: Fundação Calouste Gulbenkian; 2009.

16. Freire P. Pedagogia do deprimido. Rio de Janeiro: Paz e Terra; 1987.

17. Lucena ALR, Freitas FFQ. Ensinando e aprendendo com idosos: relato de experiência. Rev Pesquisa Cuidado é Fundamental 2016;8(2):4131-41. 